\title{
Do All Threats Work the Same Way? Divergent Effects of Fear and Disgust on Sensory Perception and Attention
}

\author{
Elizabeth A. Krusemark ${ }^{1}$ and Wen $\mathrm{Li}^{1,2}$ \\ ${ }^{1}$ Department of Psychology and the ${ }^{2}$ Waisman Center, University of Wisconsin-Madison, Madison, Wisconsin 53706
}

The extant literature indicates that threat enhances cognitive processing and physiological arousal. However, being largely based on fear-relevant processes, this model overlooks other adaptive but inhibitory mechanisms in alternative threat emotions such as disgust. Combining visual event-related potential (VERP) indices (P1 and P250/s) with a simple visual search task, we contrasted behavioral and neural responses to carefully controlled images of fear, disgust, or neutral emotion (as a baseline condition). Consistent with previous findings, fear augmented VERP amplitude and electrical current density in associate visual cortices, paralleled by facilitated object search. Conversely, disgust generated an opposite pattern of effects, reflected by reduced VERP potentials and diminished visual cortical current density along with slowed search time. These results demonstrated suppressed sensory perceptual and attentional processing of disgust information, akin to the central ecological function of disgust to minimize contact with contagious objects to avoid contamination and disease. Notably, the rapid emergence of discrimination between fear and disgust as early as 96 ms after stimulus emphasizes the efficiency of emotional classification not only between threat and nonthreat, but also within the threat domain itself. Finally, a positive correlation between anxiety and behavioral and neural divergence of fear and disgust further indicates that despite their convergence on the core affect of threat, disgust and fear instigate distinct response profiles, providing novel insights into the manifold and sometimes paradoxical symptomology in anxiety disorders.

\section{Introduction}

Varieties of animal behaviors notwithstanding, the ultimate objective of these actions is to minimize threat to ensure survival. How an organism analyzes and responds to threat remains a central question in affective sciences. To date, research has illustrated that threat heightens information processing and physiological arousal (LeDoux, 2000; Dolan, 2002; Phelps, 2006). Nevertheless, threat-related processing is oversimplified in this literature with its almost exclusive focus on the emotion of fear. This restricted scope not only has led to limited knowledge of other threat emotions such as anger and disgust (McNally, 2002), but may have yielded a partial depiction of threat and its behavioral impact.

Disgust has long been recognized as a basic emotion (Darwin, 1872) and is evident as early as in neonates in the form of distaste, becoming associated with disease avoidance and ideational revulsion later in life (Rozin and Fallon, 1987; Cisler et al., 2009). Like fear, disgust represents a central threat emotion in psychopathology, underlying distress and avoidant behavior to biological and psychological contamination and violation (Phillips et al., 1998; Woody and Teachman, 2000; McNally, 2002). Despite overlap-

Received Aug. 20, 2010; revised 0ct. 1, 2010; accepted Jan. 2, 2011.

Parts of this research were funded by National Institutes of Health Training Grant T32-MH018931 to E.A.K. We thank Chien-Chung Chen, Christopher Tyler, Jun Wang, Bas Rokers, and Jenny Liu for comments on stimulus preparation; Greta Leemkuil for help with data collection; Emily Cahill for assistance with EEG analysis; and Brad Postle and Stephan Boehm for thoughtful comments on the manuscript.

Correspondence should be addressed to either Elizabeth A. Krusemark or Wen Li, Department of Psychology, Brogden Hall, University of Wisconsin, Madison, W153706, E-mail: ekrusemark@wisc.edu orwenli@psych.wisc.edu. DOI:10.1523/JNEUROSCI.4394-10.2011

Copyright $\odot 2011$ the authors $\quad 0270-6474 / 11 / 313429-06 \$ 15.00 / 0$ ping ecological characteristics, fear and disgust often incite divergent mechanisms. At the physiological level, disgust tends to activate parasympathetic responses, reducing heart rate, blood pressure, and respiration (Ekman et al., 1983; Page, 1994), thereby suppressing action. Conversely, fear swings these systems in the opposite direction by stimulating sympathetic pathways, prompting fight or flight. At the cognitive level, fear purportedly elicits an immediate "stop-look-and-listen" response to orient sensory acquisition (Gray, 1987), whereas disgust provokes instant sensory rejection (Rozin and Fallon, 1987). Accordingly, universal facial expressions of fear and disgust comprise opposing biomechanical properties modulating visual and olfactory sensors with widened versus narrowed eyes and nostrils, increasing sensory intake in fear and dampening it in disgust (Susskind et al., 2008).

Therefore, despite being both threat related, fear and disgust could engage opposite mechanisms with contrasting behavioral consequences. However, it is unclear whether central processing of these two emotions indeed diverges to underlie these peripheral distinctions. Neural evidence of such divergence, especially relevant sensory perceptual correlates, would demonstrate refined categorization of threat emotions as early as in the initial feedforward sweep of processing, highlighting the adaptability of the human defense system to optimize actions to diverse dangers in the environment. Therefore, using pictures of real-life objects combined with visual event-related potential (ERP) components, primarily $\mathrm{P} 1$, which is sensitive to emotional modulation on visual perception (Mangun et al., 1993; Eimer and Holmes, 2002, 2007; Pourtois et al., 2004; Li et al., 2007, 2008a; Vuilleumier and Pourtois, 2007), we compared the neural correlates 
of sensory processing for these two emotions. Furthermore, in conjunction with a visual search task, we contrasted attentional effects of fear versus disgust and modeled their unique influences on everyday tasks such as object detection. Finally, in light of controversies concerning the roles of fear and disgust in anxiety (Thorpe and Salkovskis, 1998; Woody and Teachman, 2000), we examined the dissociation between these threat emotions as a function of anxiety, thereby providing new insight into this problem.

\section{Materials and Methods}

Participants. Forty-three students (mean age, 19.3 years; six men) were selected from 563 college students based on their scores on the Behavioral Inhibition Scale (BIS, a measure for trait anxiety) (Carver and White, 1994). The sample consisted of 22 students with the highest scores (ranging from 25 to 28) and 21 students with the lowest scores (ranging from 10 to 17), who were all right handed with normal or corrected-to-normal vision and denied a history of neuropsychological trauma and current use of psychotropic medication. (One participant with an extreme number of response omissions and another participant with technical problems during EEG recording were not included in this sample.) All participants provided informed consent to take part in the study, which was approved by the University of Wisconsin Institutional Review Board. Anxiety assessment. Two self-report measures were administered to each selected participant at the beginning of the experiment: the BIS (readministered), which consists of seven items, designed to assess a personality trait related to sensitivity to threat and proneness to anxiety (Carver and White, 1994; Li et al., 2007, 2008a); and the Beck Anxiety Inventory (BAI) (Beck et al., 1988), which is a 21 -item instrument tapping anxiety symptom severity, commonly used in clinical and research settings. Both questionnaires are rated on a 4-point scale between 0 and 3 (for the BAI) or between 1 and 4 (for the BIS), ranging from "very little" to "very much." Although participants completed the BIS earlier during the mass survey, we obtained the second set of BIS scores given its proximity to the experiment. Because BIS and BAI scores were closely correlated $(r=$ $0.46, p<0.01)$ and exhibited largely similar modulatory effects on threat processing, we combined these two measures into an anxiety composite index to reflect the general effect of anxiety. Individual effects of each anxiety measure can be found in supplemental Table 1 (available at www. jneurosci.org as supplemental material).

Stimuli. Images of natural objects were selected from the International Affective Picture Set (IAPS) (Lang et al., 2008) to convey fear (IAPS 1012, 1050, 1201, 1120, 6260, 6263; depicting snakes, spiders, and guns), disgust (1274, 7380, 9300, 9302, 9322, and two non-IAPS images; depicting roaches, feces, and vomit), or neutral $(1450,1640,1670,7010,7021$, 7025, 7030, 7035, 7045, 7053; depicting animals and artifacts) content. An independent sample $(n=11)$ made valence and arousal ratings for these images on a visual analog scale ranging from -10 (extremely unpleasant/not at all arousing) to +10 (extremely pleasant/extremely arousing). Valence ratings confirmed equivalent hedonic values for fear and disgust sets: fear: mean $(\mathrm{M})( \pm \mathrm{SD})=-7.54$ (2.31); disgust: -7.05 $(1.79) ; t_{(10)}=0.58, p=0.57$, but more negative ratings for these two sets than neutral images [1.35 (1.81); $\left.t_{(10)}<-9.56, p<0.001\right]$. Arousal ratings indicated that the highest arousal was induced by fearful images, followed by disgust images [fear: 6.65 (1.43); disgust: $2.77(0.94) ; t_{(10)}=$ $10.29, p<0.001$ ], and the least by neutral images [neutral: -2.49 (3.28); $\left.t_{(10)}=5.22, p<0.001\right]$. This group then rated the emotions of fear and disgust in the images on a scale of 0 (not at all) to 10 (extreme), which confirmed that the fear and disgust sets each elicited the intended emotions. Fear images elicited strong fear [8.51 (1.54)] but less intense disgust $\left[6.29(2.99) ; t_{(10)}=3.66, p<0.01\right]$. Likewise, disgust images evoked potent disgust $[8.45(0.92)]$ but less marked fear $\left[4.74(1.42) ; t_{(10)}=6.94\right.$, $p<0.001]$. As indicated by the mean rating scores, the two sets were matched in the intensity of their primary emotions $\left(t_{(11)}=-0.12, p=\right.$ $0.91)$. Finally, neutral images were rated low on both fear [0.66 (0.59)] and disgust $[0.39(0.58)]$ emotions, differing clearly from the emotion sets $(p s<0.001)$.

In addition, study participants provided ratings of the images at the end of each experiment on the same scale, further validating that fear and disgust images did not differ in valence [fear: -5.59 (2.65); disgust: $-5.44(2.35) ; t_{(42)}=0.49, p=0.63$ ], but were both more unpleasant than neutral images [2.05 (1.72); ts $\left.{ }_{(42)}<-15.86, p s<0.001\right]$. Similar to the pattern in the independent sample, fear images provoked the highest arousal, followed by disgust images [fear: 4.31 (2.89); disgust: 1.29 (1.90); $t_{(42)}=9.44, p<0.001$ ], and the lowest for neutral images [neutral: -3.18 $\left.(2.31) ; t_{(42)}=8.96, p<0.001\right]$. Therefore, the valence and arousal patterns were largely aligned with the nature of these emotions. Finally, participants' emotion ratings further confirmed the emotional content of the images. Like the independent sample, participants found fear images highly frightening [6.64 (2.32)] and the disgust images equally highly disgusting $\left[6.82(2.36) ; t_{(42)}=0.75, p=0.46\right]$. Importantly, these ratings were both greater than ratings for their alternate emotions [disgust rating for fear images: 3.41 (2.63); fear rating for disgust images: 3.41 (2.19), $p<$ $0.001]$, and neutral images evoked still lower fear [0.55 (2.19)] or disgust [0.28 (0.51), $p<0.001]$.

Efforts were made to match the physical properties of these images. First, we chose images similar in visual composition and equated the size of each image. Second, images were transformed to grayscale and normalized to equal luminance $\left(17 \mathrm{~cd} / \mathrm{m}^{2}\right)$ (Knebel et al., 2008). Finally, we screened images based on a wavelet analysis (Delplanque et al., 2007) to ensure that images in the three emotion categories did not differ in spatial frequency, thereby influencing early visual processing. Each image was decomposed into nine frequency bands $(512,256,128,64,32,16,8,4$, and $<2$ cycles per image), as well as into two broad frequency bandshigh ( $>16$ cycles/image) and low ( $\leq 8$ cycles/image). Energy in each band was submitted to ANOVA. Results confirmed that the chosen sets did not differ in any spatial frequency band ( $p s>0.16$ ).

Visual search task description. Participants were seated in a dimly lit room, $\sim 120 \mathrm{~cm}$ from a cathode ray tube (CRT) monitor. Each trial began with a crosshair at the center of the screen, followed by an image centrally displayed for $150 \mathrm{~ms}$, subtending a visual angle of $7.2^{\circ}$. Next, a search array was superimposed on the image for $500 \mathrm{~ms}$, comprising one horizontal bar (target) and seven vertical bars (distracters) presented in green (Fig. 1). Individuals were instructed to make a button press to indicate the quadrant where the target was located while maintaining fixation. A total of 20 search arrays were included, and the target appeared in each quadrant with equal probability. There were two blocks, each containing 150 trials from the three conditions randomly presented (totaling 100 trials per emotion).

Stimulus presentation was linked to the refresh rate $(60 \mathrm{~Hz})$ of the CRT screen, delivered using Cogent2000 software (Wellcome Department of Imaging Neuroscience) as implemented in Matlab (MathWorks). Synchronization between stimulus presentation and event trigger onset (recorded in the EEG acquisition computer) was verified by placing a photodiode at the bottom right corner of the CRT monitor sampled by the EEG acquisition computer at $2048 \mathrm{~Hz}$ : image onset consistently 
A

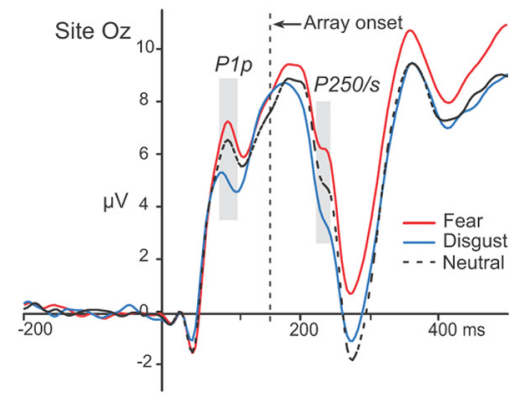

D

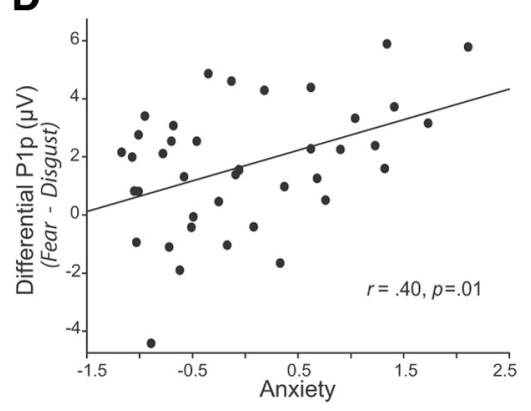

B

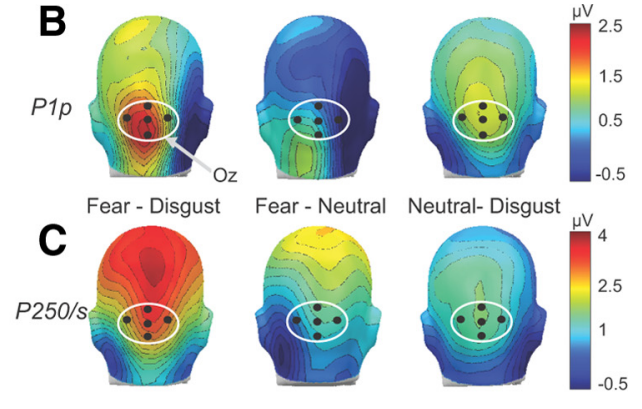

$\mathbf{E}$

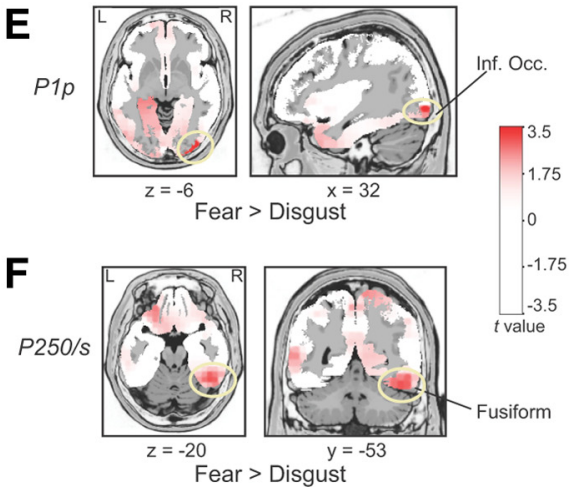

Figure 2. Opposing effects of fear and disgust on P1 and P250/s. $A$, ERP waveforms at $0 z$. B, C, Maps of differential P1p $(\boldsymbol{B})$ and P250/s ( $(\boldsymbol{C}$, with the five sensors marked with black dots, encircled to represent $0 z$. $\boldsymbol{D}$, Anxiety composite scores (anxiety severity and trait anxiety combined) positively correlated with differential P1p amplitude between fear and disgust conditions. $\boldsymbol{E}, \boldsymbol{F}$, Source estimation (LORETA) isolated the right inferior occipital gyrus exhibiting greater current density for fear than disgust conditions during the P1p interval $(\boldsymbol{E})$ and the right fusiform gyrus exhibiting greater current density for fear than disgust conditions during the P250/s interval $(\boldsymbol{F})$. Inf. Occ., Inferior occipital gyrus.

aligned with the event trigger across repeated trials $[n=40$; offset, 0.5 $(0.2) \mathrm{ms}]$.

EEG recording and analysis. EEGs were recorded from a 96-channel (BioSemi; ActiveTwo) system at a $1024 \mathrm{~Hz}$ sampling rate with a $0.1-100 \mathrm{~Hz}$ bandpass filter. Electrooculograms (EOGs) were recorded at two eye electrodes at the outer canthi and one infraorbital to the left eye. EEG signals were referenced offline to the average of the left and right mastoid recordings. Horizontal EOG channels were referenced to each other, and the vertical EOG channel was referenced to the EEG channel located directly above it. EEG/EOG signals were digitally bandpass filtered from 0.1 to $40 \mathrm{~Hz}$, downsampled to $256 \mathrm{~Hz}$, and segmented around picture onset (from -200 to $500 \mathrm{~ms}$ ). Trials with EEG/EOG voltages exceeding $\pm 75 \mu \mathrm{V}$ (relative to the $200 \mathrm{~ms}$ baseline) were excluded from analysis.

Inspection of the grand average ERP waveform indicated a P1 component (labeled here as P1p) that peaked at $96 \mathrm{~ms}$ after image onset, maximal at occipital sites (Fig. $2 A$ ). We further identified a second positive component peaking at $246 \mathrm{~ms}$ after image onset (or $96 \mathrm{~ms}$ after searcharray onset), also maximal at occipital sites. Because this component likely reflected ongoing activity to the images in addition to a P1-like response evoked by the search array, we denote this component as $\mathrm{P} 250 / \mathrm{s}$ here. Mean P1p and P250/s amplitudes were extracted at the central occipital locus (collapsed across five electrodes surrounding $\mathrm{Oz}$ ) (Fig. $2 \mathrm{~B})$ over 36 (P1p) and $28 \mathrm{~ms}$ (P250/s) intervals centered on the corresponding peak (i.e., peak \pm 4 and 3 data points, respectively, determined based on the span of these components).

Cortical sources for these ERP effects were estimated using lowresolution brain electromagnetic tomography (LORETA) (PascualMarqui et al., 1994). As an inverse solution, LORETA has been validated using neuroimaging in visual and cognitive processes (Pascual-Marqui et al., 2002). LORETA uses a three-shell spherical head model registered to standardized space from a digitized magnetic resonance imaging (MRI) at the Montreal Neurological Institute. Solutions are restricted to cortical gray matter, spanning 2394 voxels with a spatial resolution of $7 \times 7 \times 7$ $\mathrm{mm}$. Besides localizing the origin of these components, we estimated voxelwise current density during the P1p and $\mathrm{P} 250 / \mathrm{s}$ intervals for each subject in response to fear and disgust, followed by voxelwise paired $t$ tests to isolate cortical substrates for respective processes. A cluster of voxels would be identified if $t$ values in at least three neighboring voxels were significant $\left(_{(42)}>2.69, p<0.005\right.$, uncorrected).

Statistical analysis. Separate two-way repeatedmeasures ANOVAs with factors of emotion (fear, disgust, neutral images) and anxiety (as a continuous variable) were performed on search reaction time (RT) and accuracy and on the P1p and P250/s amplitude. Significant effects of emotion were followed by $t$ tests to contrast the effects of individual emotions. RTs over 2 SDs away from the mean were excluded, and remaining RTs were log-transformed before analysis to reduce skewness.

\section{Results}

\section{Behavior}

We contrasted effects of fear and disgust emotions on visual attention by examining how they influenced detection of the visual search target superimposed on the images. Emotion exerted a significant main effect on search speed $\left(F_{(2,84)}=\right.$ $12.40, p=0.001)$ (Fig. 3A). Planned comparisons indicated that RT was slowest after disgust pictures [657 (116) ms], intermediate after neutral pictures [649 (110) ms; disgust vs neutral: $t_{(42)}=3.31$, $p<0.01]$, and fastest subsequent to fearful pictures [647 (121) ms; fear vs disgust: $t_{(42)}=-4.65, p<$ 0.001; fear vs neutral: $t_{(42)}=-1.72, p=0.09$ ]. However, the emotion effect on speed did not vary with anxiety levels (composite score $)\left(F_{(2,82)}=0.72, p>0.49\right)$.

Although there was no simple effect of emotion on search accuracy $\left(F_{(2,84)}=0.08, p=0.92\right)$, it was influenced interactively by emotion and anxiety $\left(F_{(2,82)}=3.08, p=0.05\right)$ (Fig. $\left.3 B\right)$. Anxious (vs nonanxious) individuals exhibited greater accuracy in the fear than the disgust condition $(r=0.36, p<0.05)$. Together, search RT and accuracy complemented each other to demonstrate that disgust and fear emotions exerted divergent effects on visual search performance (hindrance by disgust and facilitation by fear, especially in anxious individuals).

\section{P1p response}

In parallel to the behavioral results, emotion modified the P1p amplitude $\left(F_{(2,78)}=18.38, p<0.001\right)$ (Fig. $\left.2 A, B\right)$. Fear images evoked the strongest P1 $[6.76(4.37) \mu \mathrm{V}]$, neutral images evoked an intermediate P1 [6.31 (4.43) $\mu \mathrm{V}$; fear vs neutral: $t_{(39)}=1.69$, $p=0.09]$, and disgust images evoked the weakest P1 [4.93 (4.69) $\mu \mathrm{V}$; fear vs disgust: $t_{(39)}=4.91, p<0.001$; neutral vs disgust: $\left.t_{(39)}=4.69, p<0.001\right]$.

Moreover, this emotion effect was modulated by anxiety $\left(F_{(2,76)}=4.97, p<0.01\right)$. Anxiety was positively correlated with the differential P1 between fear and disgust trials $(r=0.40, p=$ 0.01 ) (Fig. 2D). Of note, a simultaneous regression analysis of anxiety on raw $\mathrm{P} 1$ to fear images and raw P1 to disgust images indicated that both threat emotions were uniquely correlated with anxiety but in opposite directions $(s r=0.45, p<0.005$ for fear and $s r=-0.38, p<0.05$ for disgust). These results thus 
A

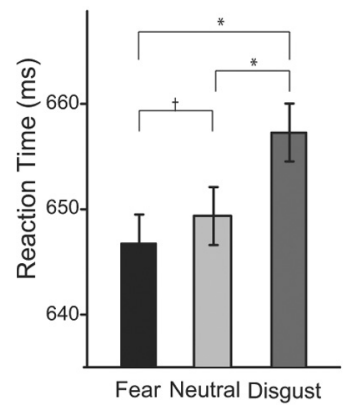

B

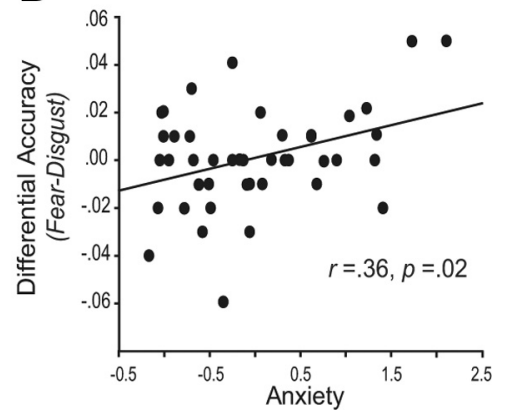

Figure 3. Opposing effects of fear and disgust on behavior. $A$, Faster search response time in fear and slower in disgust. $B$, Anxiety composite scores positively correlated with task accuracy difference between fear and disgust conditions. ${ }^{*} p<0.01,{ }^{\dagger} p<0.10$. Error bars indicate SEM.

suggest that fear and disgust pose contrary effects on early visual processing, escalating with increased anxiety.

\section{$\mathrm{P} 250 / \mathrm{s}$ response}

P250/s demonstrated opposite effects of disgust and fear on visual attention at $250 \mathrm{~ms}$ after the image (96 ms after search array) $\left(F_{(2,78)}=20.64, p<0.001\right)$ (Fig. $\left.2 A, C\right)$. Fear augmented P250/s $[5.01(6.11) \mu \mathrm{V}]$ compared to neutral pictures [3.86 (6.01) $\mu \mathrm{V}$; $\left.t_{(39)}=3.68, p=0.001\right]$. By contrast, disgust diminished P250/s $[2.34(6.23) \mu \mathrm{V}]$ relative to neutral $\left[t_{(39)}=-3.05, p<0.005\right]$ and fear $\left[t_{(39)}=-5.84, p<0.001\right]$ pictures. Unlike the P1p effect, however, this emotion effect on P250/s did not appear to significantly covary with anxiety (Fs $<1.53, p s>0.23$ ).

\section{LORETA source analyses}

Using LORETA, we first isolated the origin of P1p and P250/s (pooled across the three emotions). P1p and P250/s were both localized to the extrastriate cortex, maximal in the left lingual gyrus (P1p and P250/s: x, $y, z=-10,-95,-13$ ) (supplemental Fig. 1, available at www.jneurosci.org as supplemental material), thereby validating the identification and measurement of these early visual potentials.

Next, we searched for the neural substrates underlying divergent visual responses between fear and disgust. Voxelwise $t$ tests for the P1p window revealed a cluster of voxels in the right inferior occipital gyrus (three voxels, peaking at $32,-95,-6 ; t_{(42)}=$ $3.51, p<0.005$ ) (Fig. 2E), exhibiting greater current density in response to fear than disgust pictures. Breaking the sample into high- and low-anxiety severity groups, we found that this cluster emerged in the high-anxiety severity group alone $(32,-95,-6$; $\left.t_{(21)}=3.13, p<0.005\right)$, in keeping with the scalp P1p effects reported above. In fact, no cluster survived the preset threshold in the low-anxiety group. For the P250/s interval, voxelwise $t$ comparisons uncovered a cluster of three voxels in the right fusiform gyrus $\left(46,-53,-20 ; t_{(42)}=2.93, p<0.005\right)$ (Fig. $\left.2 F\right)$, responding more strongly after fear than after disgust pictures. In summary, these source analysis results confirmed that disgust and fear evoked differential activity in associative visual areas, thus providing further evidence of their distinct impact on early visual sensory processing and visual attention.

\section{Control experiment: control of low-level influences on early visual processes}

Previous research illustrates that early visual ERP components are sensitive to physical stimulus properties such as luminance (Johannes et al., 1995; McCourt and Foxe, 2004; Knebel et al.,
2008) and spatial frequency (Singh et al., 2000; Pourtois et al., 2005; Delplanque et al., 2007). It is therefore possible that variability in the physical properties of the picture sets might have elicited differential visual activity. Our careful selection and extensive processing of the images have largely eliminated this potential confound. Our findings that these differential responses to the picture sets were coupled with individual differences in anxiety accentuated emotional effects on P1, affording further exclusion of this possibility. To further safeguard against this confound, we conducted a control ERP experiment to assess P1 to scrambled versions of the images used in the study. Critically, by randomizing only the phase component of Fourier-transformed images, we rendered the images unintelligible without altering the Fourier energy (Chen et al., 2007), in an effort to minimize deviation from the original images.

\section{Participants and procedure}

Twelve participants were recruited (five men; mean age, 19.1 years) through the undergraduate psychology research pool at the University of Wisconsin-Madison. In each trial, a scrambled image was presented on a CRT monitor for $400 \mathrm{~ms}$, subtending a visual angle of $7.2^{\circ}$. The image was followed by a centrally presented arrow pointing left or right $(<,>)$ for $400 \mathrm{~ms}$, to which individuals were instructed to make a button press to indicate the direction of the arrow while maintaining fixation. This simple task was designed to sustain subjects' concentration on the task. Each intertrial interval consisted of a central fixation cross presented for 1000-2000 ms. The task consisted of two consecutive blocks of 150 trials.

\section{$P 1$ response to scrambled images}

ERP recording, artifact and trial exclusion, and $\mathrm{P} 1$ analyses were conducted using the same parameters as the main experiment. An ANOVA test revealed that the image set did not significantly influence P1 magnitude $\left(F_{(2,22)}=2.14, p=0.17\right)$, nor was the P1 pattern similar to that observed for the intact emotional images. P1 amplitudes were 7.05 (5.03) $\mu \mathrm{V}$ for scrambled fear images, $6.66(5.42) \mu \mathrm{V}$ for scrambled disgust, and 6.27 (5.27) $\mu \mathrm{V}$ for scrambled neutral images. We note that the clear reduction of $\mathrm{P} 1$ response to disgust pictures from that to neutral pictures in the main experiment was no longer present with the scrambled pictures.

\section{Discussion}

Using neutral images to provide a putative baseline, we demonstrated opposite impacts of fear and disgust on sensory perception and attention, paralleled by contrasting behavioral profiles toward these two threat emotions. Replicating neural and behavioral facilitation by fear in the extant threat research, our data constitute some of the first evidence of suppressed neural processing combined with impeded behavioral performance to disgust-relevant threat, affording a new dimension to the current literature. Furthermore, this functional disparity between fear and disgust is especially prominent among anxious individuals. Given the ambiguous role of disgust in anxiety and scanty research in this aspect, the demonstration of distinct processing of disgust that is especially pronounced in anxiety could pose pivotal implications in the theorization and intervention of anxiety disorders.

It is noteworthy that by presenting images of real-life objects rather than images of facial expressions, we directly assayed evoked emotional states of fear and disgust without engaging extraneous processes such as social cognition and communica- 
tion of emotion. To control for low-level physical differences in the pictures, which could bias $\mathrm{P} 1$ responses, we carefully matched image sets for basic visual properties. Moreover, our control experiment using scrambled versions of the images (phasescrambled, unintelligible images) (Chen et al., 2007) indicated no significant set effect on P1 response, further excluding this critical confound. Given that the P1 and behavioral results were incompatible with arousal ratings for the images, we also excluded arousal-related global attentional influences that could contribute to these effects. Finally, we rejected trials with eye or facial muscle movement to eliminate facial alteration of visual exposure that could contaminate the data.

The P1 to these emotional images differed as early as $96 \mathrm{~ms}$ after stimulus. This early discrimination is remarkable, even preceding the amygdala differentiation of fear versus nonthreat stimuli (Oya et al., 2002; Krolak-Salmon et al., 2004), albeit amygdala may still be critical for maintaining stable threat representation and encoding in the sensory cortex (Rotshtein et al., 2010). Notably, diminished response to disgust cannot simply be attributed to a lack of response to disgust stimuli. Rather, the clear departure of disgust-induced P1 from the neutral (baseline) condition highlighted substantial (though inhibitory) activity in the visual cortex to represent the specific state of disgust. Expanding on previous reports restricted to threat-versus-nonthreat comparisons (Pourtois et al., 2004; Vuilleumier and Pourtois, 2007), these data suggest that sophisticated visual categorization within the class of threat could occur during the initial feedforward sweep of sensory processing. Such early perceptual discrimination of emotion corroborates the notion that emotion perception begins as soon as environmental input reaches the sensory system (Barrett and Bar, 2009). Furthermore, it raises the possibility that codes of threat emotions are stored in the sensory system to support instantaneous classification (Weinberger, 2007; Li et al., 2008b; Barrett and Bar, 2009; Pessoa and Adolphs, 2010), initiating distinct downstream operations, mobilizing quick and threat-specific actions to maximize ecological benefit.

Neural and behavioral data from the visual search task may exemplify such a mechanism: swift sensory discrimination of disgust and fear directly modulated deployment of visual attention in an ongoing task, inhibiting or augmenting attention (as indexed by P250/s magnitude) with consequent delay or acceleration in search performance. Critically, these processes operated in accordance with their individual biological functions. Heightened attention and facilitated task performance conform to the well established role of fear in boosting information processing (LeDoux, 2000; Dolan, 2002; Phelps, 2006). By contrast, disgust images suppressed visual attention to the degree that they impeded search of the target in the overlapping visual field. Despite a small behavioral cost, subjects were nonetheless able to successfully divert (sensory) attention from the source of disgust, maintaining the goal of minimizing exposure to and contagion of threats such as poison and germs (Rozin and Fallon, 1987). Therefore, fear and disgust not only activate discrete sensory representations, but also orient subsequent cognitive processes and behaviors in a manner congruent to their evolutionary purposes.

As indicated by the strong correlation between anxiety and P1p magnitude and between anxiety and search accuracy, sensory and behavioral discrepancy between disgust and fear is to an extent amplified by anxiety. The direct contrast between disgustand fear-related processing in anxiety accentuates the fact that although anxiety enhances threat sensitivity and responsivity in general (Lang et al., 2000; McNaughton and Gray, 2000), the profiles for individual threat emotions could be vastly different.
In the anxiety literature, fear and disgust are often intertwined in anxious responses such that disgust is posited as an epiphenomenon-merely subdued fear (Thorpe and Salkovskis, 1998; McKay, 2002). Insofar as anxiety intensifies excitatory processing in fear and inhibitory processing in disgust, qualitative rather than quantitative differences between fear and disgust processing in anxiety evince that disgust stands as a discrete emotional state in its own right. The implication is that disgust not only intersects with fear in anxiety, but also interacts with fear (Woody and Teachman, 2000; Cisler et al., 2009). The dynamic composition of opposing mechanisms elicited by fear and disgust may account for the multifaceted and sometimes contradictory symptomology of anxiety disorders, varying widely from hypervigilance to fainting and from obsession to amnesia, that cannot be explained simply by the influence of fear or disgust alone. Accordingly, innovative therapies targeting both disgust and fear responses should hold great promise for improvement in anxiety treatment.

In affective sciences, distinctions are traditionally drawn between threat and nonthreat processing or between positive and negative stimuli. However, our findings bring to light remarkable divergence within the domain of threat in terms of sensory analysis, impacts on cognitive processes, and eventually, behavioral consequences; this underscores the necessity to conceptualize threat beyond a unidimensional system defined exclusively by physiological and behavioral amplification. In all, the current data reaffirm the incredible diversity and plasticity of biological responses, an extraordinary feat of evolution whereby refined and quick computation is achieved such that an optimal action is used to counter the enemy.

\section{References}

Barrett LF, Bar M (2009) See it with feeling: affective predictions during object perception. Philos Trans R Soc Lond B Biol Sci 364:1325-1334.

Beck AT, Epstein N, Brown G, Steer RA (1988) An inventory for measuring clinical anxiety: psychometric properties. J Consult Clin Psychol 56:893-897.

Carver CS, White TL (1994) Behavioral inhibition, behavioral activation, and affective responses to impending reward and punishment: the BIS BAS scales. J Pers Soc Psychol 67:319-333.

Chen CC, Kao KLC, Tyler CW (2007) Face configuration processing in the human brain: the role of symmetry. Cereb Cortex 17:1423-1432.

Cisler JM, Olatunji BO, Lohr JM (2009) Disgust, fear, and the anxiety disorders: a critical review. Clin Psychol Rev 29:34-46.

Darwin C (1872/1998) The expression of the emotions in man and animals. New York: Oxford UP.

Delplanque S, N'diaye K, Scherer K, Grandjean D (2007) Spatial frequencies or emotional effects? A systematic measure of spatial frequencies for IAPS pictures by a discrete wavelet analysis. J Neurosci Methods 165:144-150.

Dolan RJ (2002) Emotion, cognition, and behavior. Science 298:1191-1194. Eimer M, Holmes A (2002) An ERP study on the time course of emotional face processing. Neuroreport 13:427-431.

Eimer M, Holmes A (2007) Event-related brain potential correlates of emotional face processing. Neuropsychologia 45:15-31.

Ekman P, Levenson RW, Friesen WV (1983) Autonomic nervous system activity distinguishes among emotions. Science 221:1208-1210.

Gray JA (1987) Psychology of fear and stress. New York: Cambridge UP.

Johannes S, Münte TF, Heinze HJ, Mangun GR (1995) Luminance and spatial attention effects on early visual processing. Brain Res Cogn Brain Res 2:189-205.

Knebel JF, Toepel U, Hudry J, le Coutre J, Murray MM (2008) Generating controlled image sets in cognitive neuroscience research. Brain Topogr 20:284-289.

Krolak-Salmon P, Hénaff MA, Vighetto A, Bertrand O, Mauguière F (2004) Early amygdala reaction to fear spreading in occipital, temporal, and frontal cortex: a depth electrode ERP study in human. Neuron 42:665-676.

Lang PJ, Davis M, Ohman A (2000) Fear and anxiety: animal models and human cognitive psychophysiology. J Affect Disord 61:137-159. 
LeDoux JE (2000) Emotion circuits in the brain. Annu Rev Neurosci 23:155-184.

Li W, Zinbarg RE, Paller KA (2007) Trait anxiety modulates supraliminal and subliminal threat: brain potential evidence for early and late processing influences. Cogn Affect Behav Neurosci 7:25-36.

Li W, Zinbarg RE, Boehm SG, Paller KA (2008a) Neural and behavioral evidence for affective priming from unconsciously perceived emotional facial expressions and the influence of trait anxiety. J Cogn Neurosci 20:95-107.

Li W, Howard JD, Parrish TB, Gottfried JA (2008b) Aversive learning enhances perceptual and cortical discrimination of indiscriminable odor cues. Science 319:1842-1845.

Mangun GR, Hillyard SA, Luck SJ (1993) Electrocortical substrates of visual selective attention. In: Attention and performance, Vol XIV (Meyer D, Kornblum S, eds), Cambridge, MA: MIT Press.

McCourt ME, Foxe JJ (2004) Brightening prospects for early cortical coding of perceived luminance: a high-density electrical mapping study. Neuroreport 15:49-56.

McKay D (2002) Introduction to the special issue: the role of disgust in anxiety disorders. J Anxiety Disord 16:475-476.

McNally RJ (2002) Disgust has arrived. J Anxiety Disord 16:561-566.

McNaughton N, Gray JA (2000) Anxiolytic action on the behavioural inhibition system implies multiple types of arousal contribute to anxiety. J Affect Disord 61:161-176.

Oya H, Kawasaki H, Howard MA 3rd, Adolphs R (2002) Electrophysiological responses in the human amygdala discriminate emotion categories of complex visual stimuli. J Neurosci 22:9502-9512.

Page AC (1994) Blood-injury phobia. Clin Psychol Rev 14:443-461.

Pascual-Marqui RD, Michel CM, Lehmann D (1994) Low resolution electromagnetic tomography: a new method for localizing electrical activity in the brain. Int J Psychophysiol 18:49-65.

Pascual-Marqui RD, Esslen M, Kochi K, Lehmann D (2002) Functional imaging with low-resolution brain electromagnetic tomography (LORETA): a review. Methods Find Exp Clin Pharmacol 24:91-95.
Pessoa L, Adolphs R (2010) Emotion processing and the amygdala: from a 'low road' to 'many roads' of evaluating biological significance. Nature Rev Neurosci 11:773-783.

Phelps EA (2006) Emotion and cognition: insights from studies of the human amygdala. Annu Rev Psychol 57:27-53.

Phillips ML, Senior C, Fahy T, David AS (1998) Disgust-the forgotten emotion of psychiatry. Br J Psychiatry 172:373-375.

Pourtois G, Grandjean D, Sander D, Vuilleumier P (2004) Electrophysiological correlates of rapid spatial orienting towards fearful faces. Cereb Cortex 14:619-633.

Pourtois G, Dan ES, Grandjean D, Sander D, Vuilleumier P (2005) Enhanced extrastriate visual response to spatial frequency filtered fearful faces: time course and topographic evoked-potentials mapping. Hum Brain Mapp 26:65-79.

Rotshtein P, Richardson MP, Winston JS, Kiebel SJ, Vuilleumier P, Eimer M, Driver J, Dolan RJ (2010) Amygdala damage affects event-related potentials for fearful faces at specific time windows. Hum Brain Mapp 31:1089-1105.

Rozin P, Fallon AE (1987) A perspective on disgust. Psychol Rev 94:23-41.

Singh KD, Smith AT, Greenlee MW (2000) Spatiotemporal frequency and direction sensitivities of human visual areas measured using fMRI. Neuroimage 12:550-564.

Susskind JM, Lee DH, Cusi A, Feiman R, Grabski W, Anderson AK (2008) Expressing fear enhances sensory acquisition. Nat Neurosci 11:843-850.

Thorpe SJ, Salkovskis PM (1998) Studies on the role of disgust in the acquisition and maintenance of specific phobias. Behav Res Ther 36:877-893.

Vuilleumier P, Pourtois G (2007) Distributed and interactive brain mechanisms during emotion face perception: evidence from functional neuroimaging. Neuropsychologia 45:174-194.

Weinberger NM (2007) Associative representational plasticity in the auditory cortex: a synthesis of two disciplines. Learn Mem 14:1-16.

Woody SR, Teachman BA (2000) Intersection of disgust and fear: normative and pathological views. Clin Psychol Sci Pract 7:291-311. 